\title{
CONVERGENCE RATES FOR TIKHONOV REGULARIZATION IN FINITE-DIMENSIONAL SUBSPACES OF HILBERT SCALES
}

\author{
HEINZ W. ENGL AND ANDREAS NEUBAUER
}

(Communicated by Richard R. Goldberg)

\begin{abstract}
The main result of this paper states how the discretization parameter and regularization parameter should be chosen in relation to the noise level in order to yield the optimal convergence rate for the Tikhonovregularized solution of an ill-posed linear operator equation in a finite-dimensional subspace in the framework of Hilbert scales. The results apply to a wide class of spline and finite-element subspaces of Sobolev scales.
\end{abstract}

1. Introduction. The most widely used method for solving linear ill-posed problems is probably "Tikhonov regularization" (see [4] for a comprehensive treatment of this method). A more flexible variant of Tikhonov regularization has been introduced in [10], namely "Tikhonov regularization in Hilbert scales": Let $H, U$ be Hilbert spaces and $A: H \rightarrow U$ a bounded linear injective operator. Let $\left(H_{s}\right)_{s \in \mathbf{R}}$ be a Hilbert scale [5], i.e., $H_{s}$ is the completion of $\bigcap_{k=0}^{\infty} D\left(T^{k}\right)$ with respect to the norm

$$
\|f\|_{s}:=\left\|T^{s} f\right\| \quad(s \in \mathbf{R}),
$$

where $T$ is an unbounded selfadjoint strictly positive definite operator on $H$. (By \| $\|$ we will, according to the context, denote either \|\|$_{0}$ in $H_{0}=H$ or the norm in $U$.)

We want to solve the equation

$$
A f=g,
$$

with $g \in R(A)$; the unique solution of (1.2) will be denoted by $f_{0}$. We assume that there is an $a>0$ such that

$$
m\|f\|_{-a} \leq\|A f\| \leq M\|f\|_{-a}
$$

holds for all $f \in H$ with suitable $M \geq m>0$ (independent of $f$ ); i.e., the norms $\|A f\|$ and $\|f\|_{-a}$ are equivalent. This implies that (1.2) is ill-posed (cf. [10]).

Thus, if instead of $g$ we have noisy data $g_{\delta} \in U$ fulfilling

$$
\left\|g-g_{\delta}\right\| \leq \delta,
$$

Received by the editors October 28, 1986.

1980 Mathematics Subject Classification (1985 Revision). Primary 45L10, 65R20.

Both authors are on leave from Universität Linz, Austria, where the second author was supported by the Austrian Fonds zur Förderung der wissenschaftlichen Forschung (project S 32/03). This paper was completed during the first author's stay at the Centre for Mathematical Analysis, Australian National University, Canberra, whose support and additional travel support from Linzer Hochschulfond (Austria) are gratefully acknowledged. 
then $A f=g_{\delta}$ will either not be solvable or its solution will not be a good approximation for $f_{0}$ even for small $\delta>0$. This observation leads to the need for regularization. The optimal convergence rates will depend on the number $q>0$ in the a priori assumption

$$
f_{0} \in H_{q},
$$

which in the case of Sobolev scales is just a smoothness assumption (with some boundary conditions) about the exact solution. For $\alpha>0$, we will denote by $f_{\alpha}^{\delta}$ the minimizer of the functional

$$
f \rightarrow\left\|A f-g_{\delta}\right\|^{2}+\alpha\|f\|_{p}^{2}
$$

over $H_{p}$, where we always assume that $p \geq 0$. This minimizer exists and is unique.

In [4], the problem of choosing the "regularization parameter" $\alpha=\alpha(\delta)$ in such a way that the convergence rate for $\left\|f_{\alpha}^{\delta}-f_{0}\right\|$ is as high as possible is discussed. In [10], the following "a priori choice" of $\alpha$ leading to the optimal convergence rate is proposed for Tikhonov regularization in Hilbert scales: Then

THEOREM 1.1 (NATTERER). Let $p \geq(q-a) / 2$ and assume $\alpha \sim \delta^{2(a+p) /(a+q)}$.

$$
\left\|f_{\alpha}^{\delta}-f_{0}\right\|=O\left(\delta^{q /(a+q)}\right) \quad \text { as } \delta \rightarrow 0,
$$

which is the best possible convergence rate (among all powers of $\delta$ ) under the assumptions (1.3)-(1.5).

PROOF. Follows from [10].

Here, by " $a \sim b$ " we mean " $a=O(b)$ and $b=O(a)$."

In [3], an "a posteriori choice" for the regularization parameter was described that leads to the optimal rate (1.7).

In [7], the regularizing effect of projection onto finite-dimensional spaces was discussed, whereas in [8], (ordinary) Tikhonov regularization was combined with projection: it was shown how to choose the mesh size in such a way that the convergence rate for the regularized solution in the corresponding finite-dimensional subspaces is the same as in the infinite-dimensional setting. In this paper, we will study the corresponding question for regularization in Hilbert scales. For Sobolev scales and $p \geq q$, a result in this direction can be extracted from the proof of Theorem 2 in [9].

2. Convergence rates. We assume that the (usually finite-dimensional) subspaces we use fulfill the following assumption, which is quite usual in this context (cf. $[1$, p. 83]).

ASSUMPTION 2.1. Let $r>k \geq p, r \geq 2 p+a$, and for all $h \in] 0,1]$ let $S_{h}$ be a subspace of $H_{k}$ with the following property: There is a constant $\tilde{C}$ (independent of $u$ and $h$ ) such that for all $u \in H_{r}$ and $h>0$,

$$
\inf \left\{\|u-\varphi\|_{k} \mid \varphi \in S_{h}\right\} \leq \tilde{C} \cdot h^{r-k}\|u\|_{r}
$$

holds.

Together with an inverse assumption (cf. [1, p. 89]), Assumption 2.1 would imply an estimate like $(2.1)$ for the sum of the $H_{k^{-}}$and the $H_{-a}$-norms. Note that 
also in [8], the estimate (3.2) in a negative Sobolev norm was crucial. However, the inverse assumption is not needed:

LEMMA 2.2. Under Assumption 2.1, there is a constant $C$ (independent of $h$ and $u$ ) such that for all $u \in H_{2 p+a}$ and $h>0$,

$$
\inf \left\{h^{-a}\|u-\varphi\|_{-a}+h^{p}\|u-\varphi\|_{p} \mid \varphi \in S_{h}\right\} \leq C h^{2 p+a}\|u\|_{2 p+a}
$$

holds.

PROOF. Using techniques from interpolation theory (cf. [6]), one shows the following: For any $v \in \mathbf{R}, w>0$ and $\theta \in] 0,1], H_{v+\theta w}=B_{\theta, 2}$, where $B_{\theta, 2}=\{x \in$ $\left.H_{v} \mid k(x, t) \cdot t^{-(\theta+1 / 2)} \in L^{2}\left(\mathbf{R}^{+}\right)\right\}$with $k(x, t):=\inf \left\{\left\|x-x_{0}\right\|_{v}+t\left\|x_{0}\right\|_{v+w} \mid x_{0} \in\right.$ $\left.H_{v+w}\right\}$; the norms $\|x\|_{v+\theta w}$, defined by (1.1), and

$$
\|x\|_{\theta, 2}:=\left[\int_{0}^{\infty} k(x, t)^{2} t^{-(2 \theta+1)} d t\right]^{1 / 2}
$$

are equivalent. This puts us into the framework of [2], from which (cf. especially (4.2) there) we can derive the estimate (2.2).

For $h \in] 0,1]$, we denote the minimizer of the Tikhonov functional in $S_{h}$ (as described in Assumption 2.1) by $f_{\alpha, h}^{\delta}$. Thus, $f_{\alpha, h}^{\delta}$ solves the regularized projected problem

$$
\left\|A f-g_{\delta}\right\|^{2}+\alpha\|f\|_{p}^{2} \rightarrow \min , \quad f \in S_{h} .
$$

We finally want to estimate $\left\|f_{\alpha, h}^{\delta}-f_{0}\right\|$. Because of Theorem 1.1, it suffices to estimate $\left\|f_{\alpha, h}^{\delta}-f_{\alpha}^{\delta}\right\|$. We will do this by estimating $\left\|f_{\alpha, h}^{\delta}-f_{\alpha}^{\delta}\right\|_{p}$ and $\left\|f_{\alpha, h}^{\delta}-f_{\alpha}^{\delta}\right\|_{-a}$ and then using the interpolation inequality. The estimates will involve $\left\|f_{\alpha}^{\delta}\right\|_{2 p+a}$, which we estimate as follows.

LEMMA 2.3. Let (1.3)-(1.5) hold and let $p \geq(q-a) / 2$. Then for any $\alpha, \delta>0$, $f_{\alpha}^{\delta} \in H_{2 p+a}$. Furthermore,

$$
\left\|f_{\alpha}^{\delta}\right\|_{2 p+a}=O\left(\frac{\delta}{\alpha}+\alpha^{(q-2 p-a) / 2(a+p)}\right)
$$

as $\alpha, \delta \rightarrow 0$.

PROOF. Since (cf. $[\mathbf{1 0}$, p. 35])

$$
\left(A^{*} A+\alpha T^{2 p}\right) f_{\alpha}^{\delta}=A^{*} g_{\delta}
$$

and $A^{*} g_{\delta} \in R\left(A^{*}\right)=R\left(\left(A^{*} A\right)^{1 / 2}\right)=H_{a}$ (because of (1.3)), $f_{\alpha}^{\delta} \in H_{2 p+a}$. With $B:=A T^{-p}$ we obtain (cf. [10]) that $f_{\alpha}^{\delta}=T^{-p}\left(B^{*} B+\alpha I\right)^{-1} B^{*} g_{\delta}$ and hence

$$
\begin{aligned}
\left\|f_{\alpha}^{\delta}\right\|_{2 p+a} & \leq\left\|T^{p+a}\left(B^{*} B+\alpha I\right)^{-1} B^{*}\right\| \cdot \delta \\
& +\left\|T^{p+a}\left(B^{*} B+\alpha I\right)^{-1}\left(B^{*} B\right)^{1-(p-q) / 2(a+p)}\right\| \cdot\left\|\left(B^{*} B\right)^{(p-q) / 2(a+p)} T^{p} f_{0}\right\|
\end{aligned}
$$

(note that $B T^{p} f_{0}=g$ and that $\left(B^{*} B\right)^{(p-q) / 2(a+p)} T^{p} f_{0} \in H ;$ cf. (1.5) and $[10$, (2.2)]). 
It follows from [10, Proposition 1] (applied for $B$ with $\nu=-1$ ) that

$$
\begin{aligned}
\left\|T^{p+a}\left(B^{*} B+\alpha I\right)^{-1} B^{*}\right\| & =\left\|\left(B^{*} B+\alpha I\right)^{-1} B^{*}\right\|_{p+a} \\
& =O\left(\left\|\left(B^{*} B\right)^{-1 / 2}\left(B^{*} B+\alpha I\right)^{-1} B^{*}\right\|\right)=O(1 / \alpha) .
\end{aligned}
$$

Analogously,

$$
\begin{aligned}
\| T^{p+a} & \left(B^{*} B+\alpha I\right)^{-1}\left(B^{*} B\right)^{1-(p-q) / 2(a+p)} \| \\
& =O\left(\left\|\left(B^{*} B\right)^{1 / 2-(p-q) / 2(a+p)}\left(B^{*} B+\alpha I\right)^{-1}\right\|\right) \\
& =O\left(\alpha^{(q-2 p-a) / 2(a+p)}\right)
\end{aligned}
$$

as one sees if one expresses the operator as an integral with respect to the spectral family generated by $B^{*} B$.

These estimates imply (2.4).

Note that in general, the right-hand side of (2.4) will not go to 0 as $\alpha \rightarrow 0$ under the conditions needed in Theorem 1.1.

We are now ready to prove our main result.

THEOREM 2.4. Let (1.3)-(1.5) and Assumption 2.1 hold and let $p \geq(q-a) / 2$. Furthermore, assume that

$$
\alpha \sim h^{2(p+a)}, \quad h \sim \delta^{1 /(a+q)}
$$

holds. Then

$$
\left\|f_{\alpha, h}^{\delta}-f_{0}\right\|=O\left(\delta^{q /(a+q)}\right)
$$

as $\delta \rightarrow 0$.

Proof. First, note that (2.6) implies that $\alpha \sim \delta^{2(p+a) /(a+q)}$, so that (1.7) holds. Thus, it suffices to show that

$$
\left\|f_{\alpha, h}^{\delta}-f_{\alpha}^{\delta}\right\|=O\left(\delta^{q /(a+q)}\right)
$$

as $\delta \rightarrow 0$.

Since otherwise our estimates are only changed by positive multiplicative constants, we may assume that equality holds in (2.6) and that $m=M=1$ in (1.3). It follows from the definitions of $f_{\alpha}^{\delta}$ and $f_{\alpha, h}^{\delta}$ (cf. [4, p. 76]) that for all $\varphi \in S_{h}$,

$$
\left\|A\left(f_{\alpha, h}^{\delta}-f_{\alpha}^{\delta}\right)\right\|^{2}+\alpha\left\|f_{\alpha, h}^{\delta}-f_{\alpha}^{\delta}\right\|_{p}^{2} \leq\left\|A\left(\varphi-f_{\alpha}^{\delta}\right)\right\|^{2}+\alpha\left\|\varphi-f_{\alpha}^{\delta}\right\|_{p}^{2}
$$

and hence

$$
\left\|f_{\alpha, h}^{\delta}-f_{\alpha}^{\delta}\right\|_{-a}=O\left(\left\|\varphi-f_{\alpha}^{\delta}\right\|_{-a}+h^{p+a}\left\|\varphi-f_{\alpha}^{\delta}\right\|_{p}\right)
$$

and

$$
\left\|f_{\alpha, h}^{\delta}-f_{\alpha}^{\delta}\right\|_{p}=O\left(h^{-p-a}\left\|\varphi-f_{\alpha}^{\delta}\right\|_{-a}+\left\|\varphi-f_{\alpha}^{\delta}\right\|_{p}\right)
$$

Taking the infimum over $\varphi \in S_{h}$ and using (2.2), we obtain

$$
\left\|f_{\alpha, h}^{\delta}-f_{\alpha}^{\delta}\right\|_{-a}=O\left(h^{2(p+a)}\right) \cdot\left\|f_{\alpha}^{\delta}\right\|_{2 p+a}
$$

and

$$
\left\|f_{\alpha, h}^{\delta}-f_{\alpha}^{\delta}\right\|_{p}=O\left(h^{p+a}\right)\left\|f_{\alpha}^{\delta}\right\|_{2 p+a}
$$


Using the interpolation inequality

$$
\|x\|_{\theta(-a)+(1-\theta) p} \leq\|x\|_{-a}^{\theta}\|x\|_{p}^{1-\theta} \quad(0 \leq \theta \leq 1)
$$

with $\theta=p /(a+p)$, we obtain from (2.9), (2.10) and (2.4) (together with (2.6)) that (2.8) holds.

Thus, with the choice of the discretization parameter as in (2.6), we obtain the same rate of convergence as in the infinite-dimensional case (Theorem 1.1), which is the optimal rate under the assumptions (1.3)-(1.5). In terms of the discretization parameter, we obtain

$$
\left\|f_{\alpha, h}^{\delta}-f_{0}\right\|=O\left(h^{q}\right)
$$

which is best possible under assumption (1.5), as can be seen from [2, (4.2)].

Note that (2.6) says that if a higher order of regularization is used (i.e., $p$ becomes larger) and/or the problem becomes more ill-posed (i.e., $a$ becomes larger), then the discretization parameter should be permitted to tend to 0 more slowly in comparison with the regularization parameter (cf. [8]).

If we choose $\left.\left.T=\left(A^{*} A\right)^{-1}, p=0, a=\frac{1}{2}, q \in\right] 0,1\right]$, we obtain results for ordinary Tikhonov regularization (cf. $[10,3]$; note that Theorem 1.1 holds for $p \geq q / 2-a$ then). Condition (1.5) reduces to the usual condition

$$
f_{0} \in R\left(\left(T^{*} T\right)^{q}\right)
$$

the optimal choices (2.6) are then

$$
\alpha \sim h \sim \delta^{2 /(1+2 q)},
$$

leading to the (optimal) convergence rate

$$
\left\|f_{\alpha, h}^{\delta}-f_{0}\right\|=O\left(\delta^{2 q /(1+2 q)}\right) .
$$

Note that Assumption 2.1 is fulfilled for a wide variety of spline and finite-element spaces, for which our results can be used. E.g., in the scale of Sobolev spaces on $[0,1]$, splines on a quasi-uniform partition (with maximal distance $h$ of knots) that are piecewise polynomials of degree $2 p+a$ and overall $p$ times continuously differentiable (where $p$ and $2 p+a$ have to be replaced by the next integer if they are not integers) can be taken as elements of $S_{h}$.

\section{REFERENCES}

1. I. Babuška and A. Aziz, Survey lectures on the mathematical foundation of the finite element method, The Mathematical Foundations of the Finite Element Method with Applications to Partial Differential Equations (A. Aziz., ed.), Academic Press, New York, 1972, pp. 3-359.

2. J. H. Bramble and Ridgway Scott, Simultaneous approximation in scales of Banach spaces, Math. Comp. 32 (1978), 947-954.

3. H. W. Engl and A. Neubauer, Optimal discrepancy principles for the Tikhonov-regularization of integral equations of the first kind, Constructive Methods for the Practical Treatment of Integral Equations (G. Hämmerlin and K. H. Hoffmann, eds.), Birkhäuser, Basel, 1985, pp. 120-141.

4. C. W. Groetsch, The theory of Tikhonov regularization for Fredholm equations of the first kind, Pitman, Boston, 1984.

5. S. G. Krein and J. I. Petunin, Scales of Banach spaces, Russian Math. Surveys 21 (1966), 85-160.

6. J. L. Lions and E. Magenes, Nonhomogenous boundary value problems and applications, vol. I, Springer-Verlag, Berlin and New York, 1972. 
7. F. Natterer, Regularisierung schlecht gestellter Probleme durch Projektionsverfahren, Numer. Math. 28 (1977), 329-341.

8. __ The finite element method for ill-posed problems, RAIRO Anal. Numér 11 (1977), 271278.

9. __ On the order of regularization methods, Improperly Posed Problems and Their Numerical Treatment (G. Hämmerlin and K. H. Hoffmann, eds.), Birkhäuser, Basel, 1983, pp. 189-203.

10. $29-37$.

Institut FÜr MAthematik, UniVersität Klagenfurt, A-9022 Klagenfurt, AUSTRIA

Department of Mathematical Sciences, University of Cincinnati, CincinNATI, OHIO 45221

Current address (both authors): Institut für Mathematik, Universität Linz, A-4040 Linz, Austria 